\title{
COMPARISON OF SYSTEM METHODS (TABLE FORM) WITH SEMI SYSTEMS FROM COST ASPECTS IN THE APARTMENT MEIKARTA PROJECT
}

\author{
Anjas , HANDAYANI \\ Faculty of Engineering, Mercu Buana University Jakarta, Indonesia \\ anjashandayani@yahoo.com \\ Komarul, UMAM \\ Faculty of Engineering , Mercu Buana University Jakarta, Indonesia \\ comaroel1@gmail.com
}

\begin{abstract}
Meikarta Apartment Project at the beginning of the implementation of the method that is used is a formwork system so that the spring mounting groove formwork and casting becomes inhibited. With some consideration, the formwork method was changed from the Semi si stem method to the System (Table Form). Therefore an analysis of costs and time in formwork planning is carried out in order to obtain effective and efficient planning results. At this stage begins with field observations, literature studies include information gathering, problem formulation activities, primary and secondary data and data analysis processing with the Analysis of SNI Work Unit Price (AHS-SNI) 2018 To produce the price of formwork work which includes wages, materials and tool. Based on the results of the analysis carried out on each formwork method, it was concluded that the Cost of Formwork System Work (Table Form) is cheaper compared to Semi system. The total cost of work using the Semi system as much as Rp. 4,837,286,252.27 While using formwork System (Table Form) as much as Rp. 3,724,594,795.77. This shows that work using the System (Table Form) is cheaper by Rp. $1,112,691,456.50$ or around $23 \%$.
\end{abstract}

keywords: Semi Formwork System ; Formwork System (Table Form); Tool; Cost; Time

\section{INTRODUCTION}

The development of technology in the world of construction in Indonesia was marked by the growing number of innovations that are used in the process of construction. The role of technology grows even greater, especially to simplify the process that occurs in a construction project. One of the examples of the application of technology in the process of construction is the technology of mold concrete or formwork.

Formwork is a temporary structure because up to a certain time limit will be demolished, while the concrete structure is a permanent structure. $\mathrm{M}$ de-scribes that the structures while is a means of liaison between the design and execution of construction . The structure of the structure permanently not be built without the structures while the .

A formwork construction must meet the requirements of strength, rigidity, and stability. This requirement must be met considering that formwork is a work that is done repeatedly in high rise buildings and requires a large cost to make it ( American Concrete Institute ). The cost for formwork ranges from $40 \%-60 \%$ of the cost of concrete works or about $10 \%$ of the total building construction cost.

When this in Indonesia there are 3 types of formwork are formwork conventional, semi- systems and systems. Selection of the type of formwork is a decision that is important to the project of building multi-storey as affect the cost, time of work and the quality of construction. 


\section{METHODOLOGY}

The analysis is done based on the data of secondary that was taken from the location of the research is the Project Apartment Meikarta, with drip emphasis on the problems of the methods, the cost for the work formwork. The results of the analysis can later be used as a reference to evaluate where the system will be used for further projects . In making the analysis of the price of the unit, we must make an analysis coefficient of materials and wages, terms this is done to seek the capacity of materials and wages for 1 $\mathrm{m} 2$ her. For wages the coefficient is 1 but the price of wages is made into $1 \mathrm{~m} 2$ unit price as well. Specify material and wages are also divided into two kinds, namely materials and wages unloading / tide formwork as well as the materials and wages fabrication formwork. After we calculate the need Cost tool, Cost of materials, and the Cost of wages then the next we sum. The results are already summed multiplied by the volume of work, then we could see the price of a unit of work in total in the rupiah. Both of the tools of conventional and tool systems how is equal.

Table 1. The cost of wages and materials Install and Uninstall Formwork Plate Project Apartment Meikarta

\begin{tabular}{|c|c|c|c|c|}
\hline \multicolumn{2}{|r|}{ Description } & Coefficient & Unit Price ( $R p)$ & Total ( Rp ) \\
\hline \multirow{3}{*}{ Wage } & Formwork Settings & 1 & Rp.42,960 & Rp.42,960 \\
\hline & Dismantling Formwork & 1 & Rp10,688 & Rp10,688 \\
\hline & Unloading Install Terminal & 0.00195 & Rp. 192,900 & Rp. 376 \\
\hline \multicolumn{3}{|c|}{$\operatorname{Total}(\mathrm{A})$} & \multicolumn{2}{|c|}{ Rp. 54,023} \\
\hline \multirow{5}{*}{ material } & $7 \mathrm{~cm}$ nails & 0.089 & IDR 16,000 & $\mathrm{Rp} 1,424$ \\
\hline & Mold Oil & 0.05 & Rp. 17,500 & Rp.875 \\
\hline & Playwood / Phenolic 12mm & 0.3359 & Rp.295,000 & Rp99,091 \\
\hline & Torx Screw & 8 & Rp.340 & $\mathrm{Rp} 2,720$ \\
\hline & Torx Bird & 0.0175 & Rp10,000 & Rp175 \\
\hline \multicolumn{3}{|c|}{ Total (B) } & \multicolumn{2}{|c|}{ Rp 104,110} \\
\hline \multicolumn{3}{|c|}{ Total $(A+B)$} & \multicolumn{2}{|c|}{ Rp 158,133} \\
\hline
\end{tabular}

( Source : AHS: SNI 2017-2018, 2019) [ $\square 7] \square$.

Table 2. Additional costs Wages and Materials Install and Uninstall Formwork Plate Project Apartment Meikarta

\begin{tabular}{|c|l|c|c|c|}
\hline \multicolumn{2}{|c|}{ Description } & Coefficient & Unit Price ( Rp ) & Total ( Rp ) \\
\hline \multirow{3}{*}{ Wage } & Formwork Settings & 1 & $R p .42,960$ & Rp.42,960 \\
\cline { 2 - 5 } & Dismantling Formwork & 1 & $R p 10,688$ & Rp10,688 \\
\cline { 2 - 5 } & Unloading Install Terminal & 0.00195 & Rp.192,900 & Rp. 376 \\
\hline \multicolumn{2}{|c|}{ Total } & Rp. 54,023 \\
\hline
\end{tabular}

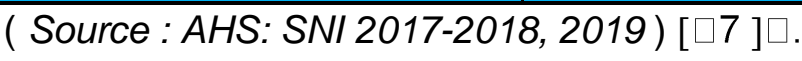

Table 3. Costs for Wages and Fabrication Materials for the Meikarta Apartment Plate Formwork Project

\begin{tabular}{|c|l|c|c|c|}
\hline \multicolumn{2}{|c|}{ Description } & Coefficient & Unit Price ( Rp ) & Total ( Rp ) \\
\hline Wage & Formwork Fabrication & 1 & 10,740 & 10,740 \\
\hline \multicolumn{2}{|c|}{ Total (A) } & \multicolumn{2}{c|}{ IDR 10,740 } \\
\hline \multirow{3}{*}{ material } & 7cm nails & 0.089 & 16,000 & 1,424 \\
\cline { 2 - 5 } & Mold Oil & 0.05 & 17,500 & 875 \\
\cline { 2 - 5 } & Playwood / Phenolic 12mm & 0.3359 & 295,000 & 99,091 \\
\hline
\end{tabular}


(International Journal of Integrated Education, Engineering Business)

\begin{tabular}{|c|c|c|c|}
\hline Torx Screw & 8 & 340 & 2,720 \\
\hline Torx Bird & 0.0175 & 10,000 & 175 \\
\hline \multicolumn{2}{|c|}{ Total (B) } & \multicolumn{2}{|c|}{$\mathrm{Rp} 104,110$} \\
\hline & & \multicolumn{2}{|c|}{ Rp 114.850} \\
\hline
\end{tabular}

( Source : AHS: SNI 2017-2018, 2019) [ $\square 7] \square$.

\section{RESULTS AND ANALYSIS}

After the unit price has been obtained, then multiplied by the volume of work. For the second floor of the first price of a unit of work post formwork can be added to the price of a unit of work fabrication but after the price which used only the price setting of the formwork. It is happening because of the use of materials formwork can be used up to four times the life.

Table 4. Price of Wage Units and Formwork Materials for Meikarta Apartments Project Plate System

\begin{tabular}{|c|c|c|c|c|}
\hline NO & FLOOR & $\begin{array}{c}\text { VOLUME } \\
\left(\mathrm{M}^{2}\right)\end{array}$ & $\begin{array}{c}\text { PRICE UNIT ( } \\
R p) \\
\end{array}$ & $\begin{array}{l}\text { AMOUNT } \\
(\mathrm{Rp})\end{array}$ \\
\hline 1 & 4th floor & $1,027.18$ & Rp.272,983 & Rp.280,402,678 \\
\hline 2 & 5th floor & $1,027.18$ & Rp.272,983 & Rp.280,402,678 \\
\hline 3 & 6th floor & $1,027.18$ & Rp.158,133 & Rp. $162,431,055$ \\
\hline 4 & 7th floor & $1,027.18$ & Rp.158,133 & Rp. $162,431,055$ \\
\hline 5 & 8th floor & $1,027.18$ & Rp. 158,133 & Rp. $162,431,055$ \\
\hline 6 & 9th floor & $1,027.18$ & Rp.158,133 & Rp.162,431,055 \\
\hline 7 & 10th floor & $1,027.18$ & Rp. 158,133 & Rp. $162,431,055$ \\
\hline 8 & 11th floor & $1,027.18$ & Rp.158,133 & Rp. $162,431,055$ \\
\hline 9 & 12th floor & $1,027.18$ & Rp. 158,133 & Rp. $162,431,055$ \\
\hline 10 & 13th floor & $1,027.18$ & Rp.158,133 & Rp. $162,431,055$ \\
\hline 11 & 14th floor & $1,027.18$ & Rp.272,983 & Rp.280,402,678 \\
\hline 12 & 15th floor & $1,027.18$ & Rp.272,983 & Rp.280,402,678 \\
\hline 13 & 16th floor & $1,027.18$ & Rp. 158,133 & Rp. $162,431,055$ \\
\hline 14 & 17th floor & $1,027.18$ & Rp. 158,133 & Rp. $162,431,055$ \\
\hline 15 & 18th floor & $1,027.18$ & Rp. 158,133 & Rp. $162,431,055$ \\
\hline 16 & 19th floor & $1,027.18$ & Rp. 158,133 & Rp. $162,431,055$ \\
\hline 17 & 20th floor & $1,027.18$ & Rp. 158,133 & Rp. $162,431,055$ \\
\hline \multicolumn{2}{|c|}{ Total Volume $\left(\mathrm{M}^{2}\right)$} & (A) & \multicolumn{2}{|c|}{$17,461.99$} \\
\hline \multicolumn{2}{|c|}{ Total Price ( $R p$ ) } & (B) & \multicolumn{2}{|c|}{$3,233,214,426$} \\
\hline \multicolumn{2}{|c|}{ Average Unit Price / $\mathrm{M}^{2}$} & $(\mathrm{~B} / \mathrm{a})$ & \multicolumn{2}{|c|}{ IDR $185,157.27$} \\
\hline
\end{tabular}

( Source : Author's Processed Data, 2019) 
Table 5. Unit Price for Wages and Formwork Material for Semi- Meikarta Apartment System Project Formation

\begin{tabular}{|c|c|c|c|c|}
\hline NO & FLOOR & $\begin{array}{c}\text { VOLUME } \\
\left(\mathrm{M}^{2}\right)\end{array}$ & $\begin{array}{l}\text { PRICE UNIT } \\
(\mathrm{Rp})\end{array}$ & $\begin{array}{l}\text { AMOUNT } \\
(\mathrm{Rp})\end{array}$ \\
\hline 1 & 4th floor & $1,027.18$ & 327,006 & $335,894,023$ \\
\hline 2 & 5th floor & $1,027.18$ & 327,006 & $335,894,023$ \\
\hline 3 & 6th floor & $1,027.18$ & 212.156 & $217,922,400$ \\
\hline 4 & 7th floor & $1,027.18$ & 212.156 & $217,922,400$ \\
\hline 5 & 8th floor & $1,027.18$ & 212.156 & $217,922,400$ \\
\hline 6 & 9th floor & $1,027.18$ & 212.156 & $217,922,400$ \\
\hline 7 & 10th floor & $1,027.18$ & 212.156 & $217,922,400$ \\
\hline 8 & 11th floor & $1,027.18$ & 212.156 & $217,922,400$ \\
\hline 9 & 12th floor & $1,027.18$ & 212.156 & $217,922,400$ \\
\hline 10 & 13th floor & $1,027.18$ & 212.156 & $217,922,400$ \\
\hline 11 & 14th floor & $1,027.18$ & 327,006 & $335,894,023$ \\
\hline 12 & 15th floor & $1,027.18$ & 327,006 & $335,894,023$ \\
\hline 13 & 16th floor & $1,027.18$ & 212.156 & $217,922,400$ \\
\hline 14 & 17th floor & $1,027.18$ & 212.156 & $217,922,400$ \\
\hline 15 & 18th floor & $1,027.18$ & 212.156 & $217,922,400$ \\
\hline 16 & 19th floor & $1,027.18$ & 212.156 & $217,922,400$ \\
\hline 17 & 20th floor & $1,027.18$ & 212.156 & $217,922,400$ \\
\hline \multicolumn{2}{|c|}{ Total Volume $\left(\mathrm{M}^{2}\right)$} & (A) & \multicolumn{2}{|c|}{$17,461.99$} \\
\hline \multicolumn{2}{|c|}{ Total Price ( $R p$ ) } & (B) & \multicolumn{2}{|c|}{$4,176,567,293$} \\
\hline \multicolumn{2}{|c|}{ Average Unit Price / $\mathrm{M}^{2}$} & $(\mathrm{~B} / \mathrm{a})$ & \multicolumn{2}{|c|}{ Rp 239,180.49 } \\
\hline
\end{tabular}

( Source : Author's Processed Data, 2019)

\section{Job Unit Price Analysis}

After we calculate the need for tools, materials, and wages, we add them up. Then we can see the price of the work unit per m2. Both of the tools of conventional and tool system . Here is a recap of the price of Rap tools, materials, and wages on the job beam and plate. Here we can see the price comparison between formwork systems and conventional formwork. 
(International Journal of Integrated Education, Engineering Business)

\section{Semi Formwork System}

Table 6. Price Works Formwork Plate Semi System Project Apartments Meikarta

\begin{tabular}{|l|c|c|c|}
\hline \multicolumn{1}{|c|}{ Description } & Volume (M⿻) & Unit Price ( Rp ) & Total ( Rp ) \\
\hline $\begin{array}{l}\text { Formwork Equipment Rental } \\
\text { Costs }\end{array}$ & 17461.99 & 32432,19 & $660,718,959.27$ \\
\hline Wage and Material Costs & 17461.99 & $239,180.49$ & $4,176,567,293$ \\
\hline \multicolumn{2}{|c|}{} & & \\
\hline \multicolumn{2}{|c|}{ Total Cost } & IDR 4,837,286,252.27
\end{tabular}

( Source : Author's Processed Data, 2019)

So , the Total Cost of Work if by using Semi System formwork is $=\mathbf{R p} . \mathbf{4 , 8 3 7 , 2 8 6 , 2 5 2 , ~} 27$

\section{Formwork System (Table Form)}

Table 7. Price Works Formwork Plate system (Table Form) for Meikarta Apartments Project

\begin{tabular}{|l|c|c|c|}
\hline \multicolumn{1}{|c|}{ Description } & Volume $\left(\mathrm{M}^{2}\right)$ & Unit Price ( Rp ) & Total ( Rp ) \\
\hline $\begin{array}{l}\text { Formwork Equipment Rental } \\
\text { Costs }\end{array}$ & 17461.99 & $28,140.00$ & $491,380,399$ \\
\hline Wage and Material Costs & 17461.99 & $185,157.27$ & $3,233,214,397$ \\
\hline \multicolumn{2}{|c|}{} & & \multicolumn{2}{c|}{ Rp 3,724,594,795.77 } \\
\hline \multicolumn{2}{|c|}{ Total Cost } &
\end{tabular}

( Source : Author's Processed Data, 2019)

So , the Total Cost of Work if by using Semi System formwork is $=\mathbf{R p} \mathbf{3 , 7 2 4 , 5 9 4 , 7 9 5 , 7 7}$

Conclusion, the Total Cost of Work using the Formwork System (Table Form) is cheaper than using the Semi System formwork worth $=\mathbf{R p} 1,112,691,456,50$

\section{CONCLUSIONS}

From the results of the analysis and discussion about the need for tools, materials, and wages and we add up, can we see the total costs are required. Both of the tools formwork Semi systems and tools formwork system (Table Form), then it can be drawn conclusions as follows :

1) After doing the calculations in Chapter IV obtained that the amount of the provision of material formwork (formwork), which takes on the system (Table Form) and Semi System as follows :

Table 8. Total supply of materials formwork Semi System and System (Table Form) Project Apartments Meikarta

\begin{tabular}{|c|l|c|c|}
\hline \multicolumn{4}{|c|}{ Comparison of Formwork Material Supply } \\
\hline No & \multicolumn{1}{|c|}{ Description } & $\begin{array}{c}\text { Spring System } \\
(\text { Pcs) }\end{array}$ & System (Table Form) (Pcs) \\
\hline 1 & Hollow 40/60 & 2268 & 2268 \\
\hline 2 & Hollow Double 50/100 & 332 & 332 \\
\hline 3 & Plat Head & - & 2408 \\
\hline 4 & U Head & 2408 & - \\
\hline 5 & Angkur U & - & 2408 \\
\hline
\end{tabular}


:: IJIEEB ::

(International Journal of Integrated Education, Engineering Business)

Volume 03 Number 02 September 2020

This work is licensed under a Creative Commons Attribution-ShareAlike 4.0 International License.

\begin{tabular}{|c|l|c|c|}
\hline \hline 6 & Angkur L and Plat strip & - & 2408 \\
\hline 7 & Vertical Support & - & 1204 \\
\hline 8 & Frame Leader & 1204 & - \\
\hline 9 & Main Frame & 1204 & - \\
\hline 10 & Horizontal Support & - & 3612 \\
\hline 11 & Cross Brace & 2364 & - \\
\hline 12 & Inner & - & 1204 \\
\hline 13 & Jack Base & 2408 & 1204 \\
\hline 14 & Caster Wheel & - & 1204 \\
\hline & Total & 12188 & 18252 \\
\hline
\end{tabular}

( Source : Author's Processed Data, 2019)

2) After doing the calculations in Chapter IV obtained that job by using the formwork system (Table Form) is saving around $20 \%-25 \%$ of beksisting Semi System at work formwork plate floor .

Table 9. Comparison of Cost Works Formwork Plate Semi System and System (Table Form) Project Apartments Meikarta

\begin{tabular}{|c|c|c|}
\hline \multirow{2}{*}{ Description } & \multicolumn{2}{|c|}{ Comparison of the cost of Formwork Work } \\
\cline { 2 - 3 } & Semi System ( Rp ) & System (Table Form)( Rp \\
)
\end{tabular}

( Source : Author's Processed Data, 2019)

Conclusion, the Total Cost of Work using the Formwork System (Table Form) is cheaper than using the Semi System formwork worth = Rp. 1,112,691,456 , 50

\section{REFERENCE}

Baharudin and Dodi, 2008. Comparative Study of the Use of Traditional Formwork and Prefabricated Formwork as Concrete Molds in High-rise Building Construction Projects, Bandung Institute of Technology.

F.Wigbout, 1997. Formwork (Printing Box). Erlangga, Jakarta.

Hanna Awad S, 1999. Concrete Formwork System, Marcel Dekker, University of Wisconsin, New York.

Mukomoko JA Ir. 1987. Basis for Building Budgeting. Primary Media Style. Jakarta.

Nemati, Kamran M. ( 2007 ) . Formwork for Concrete. Department of Constriction Management. University of Washington. Washington America .

Pamungkas, Satrio Adhi. 2013. Thesis: Job Analysis of Podium Formwork Area Using Formwork Pipe Support System and Scaffolding Formwork System (Case Study in Newton Hybrid Park Bandung Apartment Project). National Institute of Science and Technology. Jakarta

SNI 2017-2018, Unit Price Analysis of Work, Update \& Adjustment Data . 